CIENCIAS NATURALES Y EXACTAS

COMUNICACIÓN

\title{
Prácticas de innovación pedagógica, vinculación científica-tecnológica y extensión universitaria
}

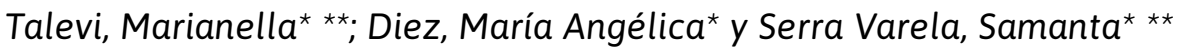

\section{Resumen}

La Universidad Nacional de Río Negro (Argentina) fortalece la vinculación con la sociedad a partir del principio de asociatividad, junto con los de ciencia, innovación y excelencia. Con enfoque cualitativo y exploratorio-descriptivo se abordaron las prácticas de innovación pedagógica en Paleontología y Geología. Estas innovaciones fueron basadas en las necesidades de los museos regionales y canalizadas a través de proyectos de extensión, voluntariado y trabajo social. Como resultado se lograron dos objetivos centrales: reactivar colaborativamente los museos hacia el interior y el exterior a través de la vinculación científico-tecnológica, mejorando la comunicación social de la ciencia y la educación científica, y enriquecer la formación de los estudiantes implementando innovaciones pedagógicas curriculares centradas en el aprendizaje-servicio. Asimismo, se fortaleció el compromiso ciudadano de los estudiantes, se prepararon las colecciones para uso de los investigadores y se generaron estrategias de preservación del patrimonio geológico-paleontológico.

Palabras clave: educación científica; comunicación científica; innovación educativa; aprendizaje colaborativo; Ciencias de la Tierra

Este trabajo fue financiado por Universidad Nacional de Río Negro, PI 40-A-794, PI 40-A-698, PI 40-A619, y la Agencia de Promoción Científica y Tecnológica (ANCyT) (PICT 0748). Recibido el 08/06/2020 y aceptado el 04/09/2020.

DOI: https://doi.org/10.33255/3161/790

Autoría: * Universidad Nacional de Río Negro. Instituto de Investigación en Paleobiología y Geología. Río Negro (Argentina). ** IIPG. UNRN. Consejo Nacional de Investigaciones científicas y Tecnológicas (Argentina).

Contacto: mtalevi@unrn.edu.ar 


\title{
Practices of pedagogic innovation, scientific-technological linkage and university extension
}

\begin{abstract}
The National university of Río Negro (Argentina) strengthens its links to society at large following the association principle, together with those of science, innovation and excellence. Innovative pedagogic practices in Paleontology and Geology were approached with a qualitative and exploratory-descriptive focus. Those innovations were based on the needs of regional museums channeled through extension projects, volunteering and social work. As a result, two central objectives were fulfilled, i.e., collaboratively reactivate museums -both inwardly and outwardly- by means of technological linking and thus improving social communication of science and science education. This, in turn, enriches student formation by implementing pedagogic curriculum innovations centered in problem solving and service-learning. Likewise, student commitment as citizens was strengthened, research collections were assembled and curated and strategies of geological-paleontological heritage preservation were generated.
\end{abstract}

Keywords: scientific education; scientific communication; educational innovation; collaborative learning; Earth Sciences

\section{Prácticas de novação pedagógica, articulação científico-tecnológica e extensão universitária}

\section{Resumo}

A Universidade Nacional de Rio Negro (Argentina) fortalece o vínculo com a sociedade com base no princípio de associatividade, junto com os de ciência, inovação e excelência. Com uma abordagem qualitativa e exploratório-descritiva, foram abordadas as práticas de inovação pedagógica em Paleontologia e Geologia. Essas inovações foram baseadas nas necessidades dos museus regionais e canalizadas através de projetos de extensão, voluntariado e trabalho social. Como resultado, dois objetivos centrais foram alcançados: reativar os museus de forma colaborativa para o interior e para o exterior através da articulação científico-tecnológica, melhorando a comunicação social da ciência e a educação científica e enriquecer a formação dos estudantes por meio da implementação de inovações pedagógicas curriculares focadas na aprendizagem-serviço. Da mesma forma, foi fortalecido o compromisso cidadão dos estudantes, as coleções foram preparadas para uso dos pesquisadores, e foram geradas estratégias de preservação do patrimônio geológico-paleontológico.

Palavras-chave: educação científica; comunicação científica; inovação educacional; aprendizagem colaborativa; ciências da Terra 


\section{Introducción}

En 1918, cuando surge La Reforma de Córdoba, desde las universidades argentinas se plantea la necesidad de fortalecer la función social de la universidad (poner al servicio de la sociedad el saber y ocuparse de los problemas de entorno) a través de la Extensión Universitaria (D’Andrea, Zubiría, y Sastre, 2012). Las actividades de vinculación, como las de extensión, se basan en un compromiso directo con la sociedad y constituyen -desde fines del siglo XX- la «tercera misión» de las universidades (Bueno Campos, 2007; Bas, 2017; Kagan y Diamond, 2019). En la década del 2000 comienza en algunos países a replantearse la idea de «transferencia de tecnología» como «transferencia de conocimientos», abarcando todas las disciplinas y ampliando las áreas de acción (Bas, 2017).

El concepto de vinculación cuenta con cierta trayectoria en las universidades en relación con la articulación con las empresas y el desarrollo productivo, aun cuando se manifiestan divergencias en sus objetivos y planificación desde los ámbitos de gestión política. La vinculación continúa siendo un territorio a explorar, definir, organizar y consolidar, cuando es con entidades no empresariales y sin fines de lucro, así como los alcances y diferencias entre vinculación tecnológica, científica o social. En ambos casos se entiende, de manera general, que la vinculación tecnológica tiene por finalidad que la sociedad o ciertos sectores de ella se vean beneficiados por la transferencia de conocimientos científicos-tecnológicos, teniendo como objetivo la resolución de problemas reales, específicos y contextualizados. Como afirmaron Rivas et al. (2014: 23), «las políticas de innovación requieren, entre otros aspectos, fomentar e impulsar capacidades en ciencia y tecnología, de modo de incentivar la cooperación y la vinculación entre el sector productivo, la academia y el sector público». Alcántar et al. (2004) destacaron la oportunidad de las universidades de atender no solo a su función de formar profesionales y a lo productivo, sino también al compromiso social, logrando otra construcción de su «imagen» $y$ «posicionamiento» en la sociedad.

El avance en los procesos de vinculación tecnológica responde a la necesidad de resolver la distancia entre la producción de nuevos conocimientos y los problemas surgidos en los ámbitos económicos o sociales (Neffa, 2001). Esto genera la creación de estructuras de interfase, que en la Argentina surgieron desde la década de 1990 como Unidades de Vinculación Tecnológica (UVT) entre el sector productivo y el sistema científico-técnico universitario. Por su parte, Rivas et al. (2014: 32) reconocieron que: 
Talevi, Marianella; et al. I Prácticas de innovación pedagógica, vinculación científica-tecnológica...

los nuevos paradigmas y desarrollo tecnológicos hacen que cada vez sea más necesaria una mayor velocidad de respuesta a las demandas del mundo productivo y los espacios de vinculación academia-Estado-empresa-sociedad civil se tornan más y más relevantes para poder avanzar por sendas de desarrollo inclusivo y sostenible.

En la vinculación tecnológica entre universidad, Estado e instituciones intermedias sin fines de lucro (e.g. museos), en que intervienen procesos educativos hacia el interior de la universidad como hacia la comunidad, resulta valioso profundizar en el modelo interactivo de innovación. Este modelo revaloriza la interacción, planteando que:

el proceso de innovación desde la concepción interactiva conduce a que lo más destacable es que todos los actores y la sociedad en general reposan sobre un conjunto de conocimientos con los cuales se interactúa constantemente, es decir se realiza un fluido intercambio con el stock existente de conocimientos al mismo tiempo que se elaboran y agregan nuevos conocimientos (Kabale, 2010: 45).

La Universidad Nacional de Río Negro (UNRN) se caracteriza, desde su creación en 2008, por un planteo de vinculación con la sociedad sustentado en el principio de asociatividad, junto con los de ciencia, innovación y excelencia. La asociatividad surgió como una estrategia para el desarrollo y la calidad, fomentando el vínculo con distintas entidades y asociaciones del entorno regional, nacional e internacional. La distribución geográfica de sus tres sedes, Atlántica, Andina y Alto Valle-Valle Medio, cada una con establecimientos en distintas localidades, muestra su inserción en toda la provincia. Esto se complementa con la integración de representantes de las empresas y de los gobiernos provincial y municipal a sus órganos colegiados de gobierno. Estas asociaciones tienen por finalidad mejorar la calidad de vida y favorecer los procesos productivos, lo cual se refleja en los planes estratégicos para el desarrollo regional de la UNRN.

En este trabajo se abordan las actividades e innovación pedagógica realizada desde las licenciaturas en Paleontología y en Geología en asociación con los museos, enmarcadas en proyectos de extensión, voluntariado o trabajo social que iniciaron cuestiones de vinculación científico-tecnológica. Los objetivos tendieron a: reactivar los museos hacia el interior (e.g. organización de las colecciones) y el exterior para mejorar su oferta educativa y cultural (e.g. material de divulgación), enriquecer la formación académica curricular de los estudiantes universitarios y su compromiso ciudadano, desarrollar inno- 
vaciones educativas en espacios externos al aula para insertar los aprendizajes en la realidad, favorecer las investigaciones mejorando la disponibilidad de las colecciones, y generar conciencia acerca del patrimonio geo-paleontológico. Se describen dos proyectos de voluntariado y dos Programas de Trabajo Social (PTS), desarrollados en asociación entre la UNRN y dos entidades regionales con colecciones paleontológicas y geológicas, los museos «Carlos Ameghino» (Cipolletti) y «Estación Cultural Lucinda Larrosa» (Fernández Oro), ambos en la provincia de Río Negro. Se contó con el compromiso de escuelas para el primer museo, y de la Municipalidad para el segundo.

Este tipo de acciones propician actividades relacionadas con la transferencia de conocimientos hacia la resolución de problemas propios del entramado sociocultural, promoviendo el intercambio de saberes y el enriquecimiento de competencias científico-tecnológicas que sirven al desarrollo y crecimiento de las organizaciones con las que se establece la vinculación. A su vez, permite a los estudiantes aplicar conocimientos adquiridos y construir nuevos, sin perder los fundamentos centrales referidos al compromiso social. Esto implica desarrollar competencias para detectar problemas locales y regionales, profundizando los conocimientos a través de las prácticas en situaciones reales, siendo fundamental que se generen soluciones socialmente responsables, integrándose para ello a las entidades y a sus actores sociales, lo que termina generando cambios curriculares. A la vez, fortalecer una formación académica que implique la construcción de una mentalidad cívica, como ciudadanos y futuros profesionales (Hatcher, Bringle y Hahn, 2017).

\section{Métodos y técnicas}

En este trabajo se abordan con enfoque cualitativo y exploratorio-descriptivo una serie de prácticas de innovación pedagógica en Geología y Paleontología que implicaron la vinculación científica-tecnológica de la universidad con las necesidades de dos museos regionales. Se recurrió al enfoque cualitativo porque se estudiaron casos concretos acotados en el espacio, tiempo e instituciones, así como a determinados grupos de estudiantes y de docentes. Así mismo, este enfoque permitió reconocer la impronta de los participantes en el desarrollo de las experiencias que implicaban involucrarse con problemas locales y regionales (perspectiva «desde adentro» no generalizable), a través de la comunicación y el aprendizaje interpelados por estrategias interactivas y dialógicas; produciendo en los estudiantes y los profesores -actuando en su doble papel de docentes e investigadores- distintas instancias de reflexión (Flick, 2004). 
Para el relevamiento de datos, información y valoración de las experiencias de los proyectos en sus etapas de avance y cierre se recurrió a cuestionarios que los estudiantes participantes respondían por escrito, así como a grupos focales. Asimismo se realizaron entrevistas a los docentes participantes, directores de museos y, en algunos casos, de las escuelas. La información fue considerada como datos primarios, en cuanto a que fueron obtenidos de la realidad que iba siendo construida con la participación de los propios investigadores, siendo relevante su observación participante, directa y no estructurada (Taylor y Bogan, 1984). Finalmente, otra fuente para la evaluación de las experiencias fue la producción académica de los estudiantes, especialmente las presentadas en jornadas y congresos (resúmenes, pósteres y exposiciones).

Posteriormente se analizaron los resultados de estos relevamientos, realizando descripciones e interpretándolos en relación con el marco institucional de la UNRN y a los modelos teóricos a los que adhiere como universidad social y emprendedora, así como en el contexto regional de esta institución y de los museos en donde se realizaron las prácticas del aprendizaje-servicio. Para ello se consideró bibliografía referida a la concepción teórica de alfabetización científico-tecnológica en la enseñanza universitaria, la comunicación social de la ciencia y la educación científica; planteos que van tomando mayor fuerza en los procesos educativos de esta universidad y que constituyen una cuestión central en el aprendizaje-servicio en las disciplinas científicas, como Geología y la Paleontología.

\section{Contexto de las experiencias y acciones realizadas}

\section{La Universidad Nacional de Río Negro y su inserción en la sociedad}

En la creación de la UNRN se expresó el interés y la colaboración del gobierno nacional, provincial y de los municipios, por los beneficios mutuos de la asociatividad, que continúa reforzándose para promover el desarrollo provincial «en línea con las potencialidades y las capacidades económicas, sociales y de IyD de la zona» (UNRN, 2016a: 29). En el Proyecto institucional (UNRN, 2009) se estableció la integración a la sociedad y el arraigo a la región, así como el compromiso de abordar sus problemáticas productivas, sociales y cultural-educativas. En la Autoevaluación institucional 2009-2015 (UNRN, 2016a: 32), se expresó el principio de asociatividad para dar respuestas a la sociedad, y para ello esta universidad «se construyó sobre la base de un modelo regional, con el propósito de atender el amplio territorio provincial mediante la aplicación de una estructura de sedes». La expansión provincial con sus múltiples localizaciones y la inclusión en su gobierno de representantes externos 
favoreció la asociatividad, fortalecida con convenios de colaboración. Estas decisiones se orientan al desarrollo interno de la ciencia y la enseñanza, así como al mejoramiento productivo y de calidad de vida (e.g. Plan estratégico 2015-2025 de Geología - UNRN, 2015).

La UNRN retoma los lineamientos estratégicos de «universidad social» y de «universidad emprendedora» planteados por B. Clarck y que Tarapuez Chamorro et al. (2012: 107) sintetizaron como social y emprendedora. Social, porque «se arroga un papel activo para la discusión y resolución de problemas de la Sociedad en la cual se inserta». Y emprendedora, porque:

tiene aspectos comunes con la empresarial pero con un matiz importante en sus objetivos; más que como un bien económico objeto de intercambio, utiliza el conocimiento como un potencial al servicio de los objetivos de su entorno socioeconómico, esto es, un recurso que (...) le permite desempeñar un papel más activo en su contexto social.

En este sentido, Pugliese (2014: 177) recalcó que «se requiere no solo mejorar la calidad y el rendimiento de la enseñanza, la investigación y la extensión, sino también orientar estratégicamente todas estas actividades en pos de objetivos institucionales y sociales claros».

Estos modelos incluyen el afán por obtener ingresos de una diversidad de fondos, no solo del Estado, lo cual genera un recorrido marcado por las relaciones y acciones conjuntas con un número mayor y más variado de actores externos. Sea por demandas internas o externas, el accionar colaborativo produce una sinergia en torno a metas, objetivos y actividades que favorecen obtener resultados innovadores. Tarapuez Chamorro et al. (2012: 108) afirmaron que:

el papel tradicional de la universidad como «simple» productora de graduados se ve dinamizado al trasladar el conocimiento fuera de sí misma mediante estrategias que le permiten la interacción entre sus actividades internas de docencia e investigación, con instituciones del medio externo con las que puede generar beneficios mutuos.

El modelo de «universidad social» le permite a la UNRN no reducir su capacidad emprendedora a las demandas del mercado y orientar proyectos que la caracterizaran como «universidad emprendedora», lo cual se refleja en los resultados obtenidos (UNRN, 2016a; 2016b). Este diseño de universidad resulta altamente complejo. La trayectoria de su primera década muestra cómo se enriquece la institución hacia adentro y en sus múltiples proyecciones hacia 
afuera, en una sinergia con lo social y lo productivo del contexto regional. Sin embargo, como se expresó en la Autoevaluación institucional 2009-2015 (UNRN, 2016b), no solo se generan beneficios sino que se acentúan las tensiones y fallas ante una diversidad de situaciones; como lo demuestran estudios de la «tercera corriente» para Reino Unido (Watson, Hal y Tazzyman, 2016; Kagan y Diamond, 2019). La UNRN:

se ve compelida a responder a la petición de amplios sectores de la sociedad, concertando diferentes propuestas, actividades y adhesiones e integrando a organismos e instituciones académicas y científico técnicas con asiento en la provincia o fuera de ella para consensuar una oferta consistente y pertinente a las necesidades de cada región o sede y al aprovechamiento de los desarrollos y recursos en I+D existentes (UNRN, 2016b: 29).

\section{Políticas de vinculación de la Universidad Nacional de Río Negro en la región a través de los Programas de Trabajo Social}

Para afianzar el vínculo entre la universidad y el sector productivo o social, es necesario generar políticas públicas que promuevan la transferencia de los conocimientos académicos y las tecnologías, dando respuestas a las demandas de dichos sectores. Desde las universidades y en relación con las denominadas sociedades del conocimiento y a las economías de bienestar, se transita un proceso de cambio del modo de ciencia I (tradicional) al II, modificando centralmente sus vínculos científico-tecnológicos con la sociedad (Gibbons et al., 1997; Arias Pérez y Aristizábal Botero, 2011). Avanzar hacia el modo II implica dar relevancia a: el contexto de aplicación en una comunidad específica (utilidad para la sociedad, el Estado y las empresas); el enfoque transdisciplinar; la heterogeneidad de los actores sociales involucrados; la organización horizontal y transitoria; la responsabilidad social inserta en el objeto de estudio y en los procesos de investigación; los criterios de evaluación que incluyan no solo la validación de la comunidad científica, sino también de la sociedad.

En materia de transferencia de conocimientos, la política general de la UNRN es brindar respuesta calificada a los problemas de la comunidad, con una visión de participación y crecimiento de la ciudadanía. Se promueve la cultura en todas sus formas; se fomenta el desarrollo tecnológico y productivo local y regional, privilegiando sectores socialmente postergados; se busca producir tecnologías y prestar servicios técnicos y de asesoramiento con proyección social, atendiendo prioritariamente las necesidades públicas y de grupos desprotegidos. 
Talevi, Marianella; et al. I Prácticas de innovación pedagógica, vinculación científica-tecnológica...

Los Programas de Trabajo Social (PTS) (UNRN, 2018a) se crearon:

con el propósito de acompañar la resolución de problemas de la comunidad, en particular de los sectores más vulnerables, compartiendo y transmitiendo conocimientos, habilidades y destrezas vinculados a la vida cotidiana y al ejercicio pleno de sus derechos y del desarrollo de la ciudadanía (Art. 77).

Así, se fomentan vínculos con la sociedad desde un enfoque ético de responsabilidad social universitaria (RSU), absorbiendo ideas y prácticas que, en las últimas décadas, generan algunas empresas en respuesta a nuevos paradigmas empresariales como Responsabilidad Social Empresarial (RSE), o Corporativa (RSC), o Estratégica (RSE) (Carrioti Peri y Schulze Lepe, 2010; Gaete Quezada, 2011). Los avances en la RSU son menores que para las empresas y resulta complejo aunar criterios en cuanto a su significado y alcances. Se advierten tres enfoques: gerencial, normativo y transformacional.

Con una visión de lo que debe ser la formación de los futuros profesionales, la UNRN convirtió la aprobación de los PTS en un requisito para la titulación. De esta manera, constituyen una instancia obligatoria, cuyo objetivo es que los estudiantes asuman un mayor compromiso social con la sociedad con proyección a su ejercicio profesional. Este cambio implicó replantear los diseños curriculares (Kagan y Diamond, 2019) ya que los PTS surgieron con posterioridad a la creación de la mayoría de las carreras y, por lo tanto, no se contemplaban en los planes de estudio; proceso en el que muchas carreras están trabajando, como Paleontología y Geología, a fin de consolidarlos como espacios curriculares.

Los PTS pueden surgir de un proyecto de extensión, voluntariado o equivalente y/o como propuestas de las cátedras o a sugerencia de las escuelas de docencia o de las unidades ejecutoras de investigación. Se conforman con docentes, estudiantes y, en algunos casos, con no docentes, y contempla la posibilidad de que los integren estudiantes de distintas carreras de una misma sede. De esta manera, se propicia la interdisciplina y se renuevan los planteos epistemológicos para pensar la integralidad de las funciones universitarias, es decir, investigación, formación y extensión (Bruno, 2016).

El PTS incluye tres componentes: formativo, comunitario y de vinculación. Estos componentes se ven proyectados en el reglamento para la aplicación los PTS (UNRN, 2018b), entre cuyos objetivos se encuentran vincular la universidad a las comunidades y su territorio para integrar sus necesidades y problemas a la labor académica generando colaboración; ofrecer a los docentes un espacio que potencie el perfil extensionista y, finalmente, que el futuro profesional 
asuma un compromiso social con el entorno en el que los saberes disciplinares se articulen con problemas sociales reales.

En el Plan estratégico 2015-2025 de Geología (UNRN, 2015) se avanzó en la consolidación del modo II, proponiendo la articulación con instituciones, empresas y reparticiones del Estado, como las dedicadas a la minería, el gas y el petróleo. Así, participaron junto con la UNRN empresas (e.g. Empresa de Desarrollo Hidrocarburífero Provincial S.A., Cámara Minera de Río Negro, YPF, Grupo Techint y Barrick Gold Argentina), organismos gubernamentales (e.g. Secretarías de Minería, de Energía y de Medio Ambiente de la provincia de Río Negro) e instituciones científicas y tecnológicas (e.g. Consejo Nacional de Investigaciones Científicas y Técnicas, y Agencia Nacional de Promoción de la Investigación, el Desarrollo Tecnológico y la Innovación). La autoevaluación concluyó que:

actuaron sinérgicamente aumentando la cooperación que permitió mejorar diferentes procesos de fortalecimiento de las capacidades de desarrollo de servicios tecnológicos para la producción en el área de la exploración y producción de hidrocarburos y la minería en el norte de la Patagonia, como así también en el campo de la Geología Ambiental. Las actividades realizadas aumentaron la inversión y redundaron en el fortalecimiento de los procesos de innovación asociativos. (UNRN, 2015: 11)

En el caso de Paleontología y en Geología de la UNRN se reconoce el modelo transformacional caracterizado por:

un fuerte compromiso social, ejecutando actividades de voluntariado, cooperación al desarrollo y acción social, con el propósito de conectar con el tejido social local mediante una comunidad universitaria conformada por personas socialmente implicadas (Gaete Quezada, 2011: 126).

\section{Interés de la sociedad por el patrimonio geo-paleontológico: Vinculación científico-tecnológica universidad y museos regionales}

En las carreras de Paleontología y de Geología de la UNRN, desde el 2010, cuando comienza la primera cohorte de alumnos, la gestión del Vicerrectorado y de los coordinadores de ambas carreras dieron prioridad a la relación con los museos. Una de las primeras medidas fue un convenio con el Museo Patagónico de Ciencias Naturales «Juan Carlos Salgado» (General Roca, provincia de Río Negro) para desarrollar docencia e investigación. La universidad estableció aulas en el museo, lo que facilitó el acceso al repositorio de rocas, minerales y 
fósiles. Como actividades prácticas de los talleres de métodos y de técnicas en paleontología y geología los estudiantes limpiaron, clasificaron y organizaron parte de esas muestras paleontológicas.

Otras experiencias se realizaron en el Museo Provincial «Carlos Ameghino» de Cipolletti (Fig.1), primero como Proyecto de Voluntariado (2011-2012) en el que se integraron los intereses de la universidad, el museo y las escuelas, del cual se desprendió un PTS (2012-2013). En ambos casos la vinculación surgió a través del director del museo, quien manifestó su preocupación ante «la situación caótica reinante en el depósito», agudizada por los problemas edilicios y la falta de personal. En las actividades de vinculación participaron docentes y estudiantes de Paleontología y de Geología y una asesora en cuestiones pedagógicas, junto al director del museo y un docente de una escuela.

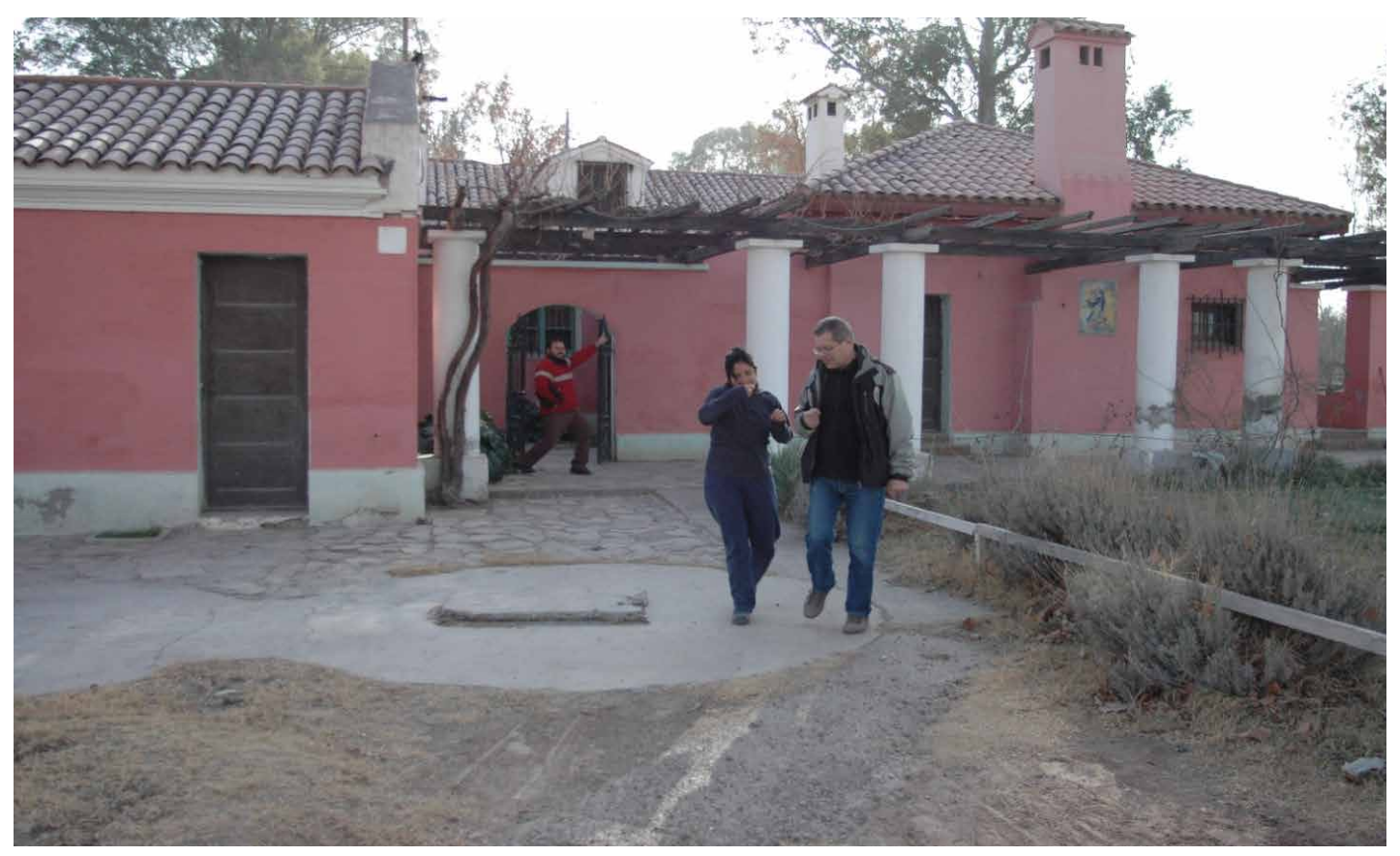

Figura 1. Museo Provincial «Carlos Ameghino», provincia de Río Negro, Argentina, donde desde la dirección junto con estudiantes y docentes de la UNRN se realizaron actividades de vinculación científico-tecnológicas (2014)

El enfoque implicaba un cambio en la cultura universitaria por cuanto solicitaba establecer la articulación con la currícula académica, es decir, reconocer el trabajo de voluntariado de los estudiantes como parte de los aprendizajes de algunas asignaturas, y contemplarlo reconocerlo para su acreditación. El replanteo también es metodológico, ya que los diseños didácticos desde las 
asignaturas deben dar la oportunidad a que los estudiantes universitarios puedan identificar un problema en la comunidad, hacer los diagnósticos y proponer las soluciones (Suárez et al., 2017). En un principio, en muchas actividades de vinculación y extensión de la UNRN no se recurría de forma explícita y reflexiva a sustentos teóricos, de ahí que no se incorporaba con claridad lo que se realizaba en el medio a los diseños curriculares, aun cuando los documentos institucionales establecieran la importancia del «aprovechamiento de los desarrollos y recursos en I+D existentes» y el reconocimiento de "las potencialidades y las capacidades económicas, sociales y de I+D de la zona» (UNRN, 2016a: 29).

Entre ambas experiencias en este museo se trabajó durante cuatro años, mucho más tiempo del previsto, porque el compromiso de los estudiantes los Llevó a continuar las actividades habiendo concluido el voluntariado y el PTS. Como resultado se logró: la recuperación y reordenamiento del primer archivo del museo que funcionaba mediante un sistema de fichas; el reconocimiento y agrupamiento, según su procedencia y fecha de extracción, de numerosos restos fósiles que hasta ese momento no estaban preparados ni estudiados; el mantenimiento de algunas piezas consideradas en estado crítico de conservación; la sistematización de los materiales paleontológicos y su ingreso a una base de datos especialmente diseñada; el registro fotográfico de cada pieza para su rápido y seguro reconocimiento; finalmente, la preparación de cuadernillos de divulgación como material didáctico para uso en las escuelas.

La siguiente experiencia la constituyeron dos trabajos de vinculación científico-tecnológica relacionados entre sí que reunieron a la UNRN, la Municipalidad y el Museo «Fernández Oro», provincia de Río Negro (Fig. 2). El primer trabajo no surgió a propuesta de la universidad, sino por una inquietud manifestada desde la Dirección del museo a docentes investigadores de la UNRN, ante la necesidad de resolver los problemas científico-tecnológicos para establecer un adecuado repositorio de los materiales. El problema central a resolver era que el museo contaba con materiales paleontológicos, geológi$\cos$ y zoológicos que no estaban correctamente identificados, clasificados y ordenados con criterios científicos; por lo tanto, no podían concebirse como colecciones. 


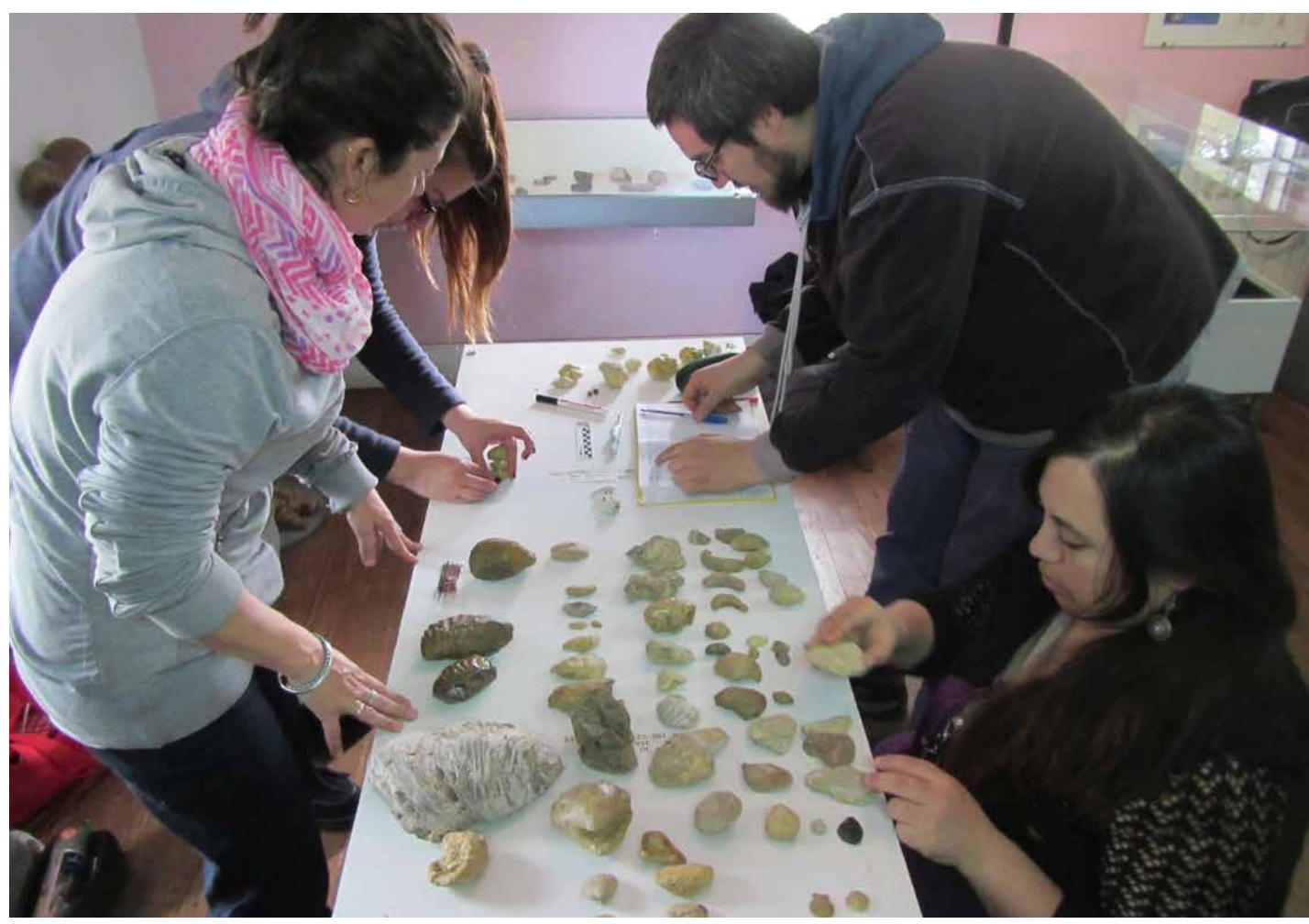

Figura 2. Estudiantes y docentes de la UNRN en actividades de vinculación científico-tecnológicas en el Museo «Fernández Oro», provincia de Río Negro, Argentina

A diferencia del caso anterior, aquí primero se desarrolló un PTS diseñado por los docentes y estudiantes de Paleontología y de Geología, con el acompañamiento del personal del museo. Posteriormente, esta experiencia fue consolidada a través de un proyecto de voluntariado universitario (2017).

El museo requería de este trabajo para: ofrecer un adecuado servicio de acceso a las colecciones por parte de los investigadores, rediseñar de la sala de exposición de Ciencias de la Tierra con objetivos educativos y abrirla a los escolares y al público. A partir de estas necesidades y de los problemas diagnosticados en los materiales del museo, se establecieron los siguientes objetivos:

1. Colaborar en la preparación y clasificación de material fósil y geológico del museo, y en el acondicionamiento de la sala de exposición de Ciencias de la Tierra.

2. Aportar a la comunidad diversos conocimientos sobre el patrimonio fósil y saberes geo-paleontológicos para su protección, cuidado y preservación.

3. Difundir en la comunidad y la región conocimientos de las Ciencias de la Tierra que son parte de la formación de los estudiantes universitarios de Paleontología y Geología. 
4. Generar conciencia en la sociedad de la necesidad de protección del patrimonio fósil.

El logro de estos objetivos tuvo como protagonistas centrales a los estudiantes universitarios participantes, puesto que la propuesta de los PTS se centró en completar la formación curricular desde la proyección de sus saberes en una comunidad específica y construir nuevos conocimientos, tanto para reforzar los aprendizajes curriculares como para fortalecer su compromiso social y cívico a través de la resolución de problemas en contextos comunitarios reales. Lo que Kagan y Diamond (2019) plantean como «activismo académico», que implicó acciones de educación científica y divulgación de la ciencia (Blanco López, 2017).

Se cumplieron en distintas etapas, definidas a partir de conocimientos científicos y técnicos, y fueron cumplidas principalmente en el museo. El Instituto de Investigación en Paleobiología y Geología (IIPG, CONICET - UNRN) constituyó el ámbito científico de respaldo, donde se desempeñan como investigadores los docentes de estos proyectos. La primera etapa consistió en el relevamiento de los materiales paleontológicos y geológicos, que incluyó identificarlos, organizarlos y clasificarlos para generar un repositorio de colecciones. Como resultado se estableció un inventario que incluyó fotografía de cada pieza, número de colección y clasificación. Así se logró valorizar y potenciar el patrimonio paleontológico, geológico y zoológico, y reactivar las funciones del museo.

En una segunda etapa, se seleccionó el material para la sala de Ciencias de la Tierra, se acondicionaron las piezas a exhibir y se le brindó conocimientos específicos sobre esas piezas al personal del museo. Además, se realizaron sugerencias pedagógicas a fin de mejorar la propuesta de este como ámbito educativo y cultural. Finalmente, los estudiantes universitarios prepararon materiales de divulgación científica de las colecciones organizadas, como transposición de los conocimientos científicos a saberes accesibles al estudiantado y al público, mejorando su servicio educativo. Además, facilita las acciones de concientización en torno a la importancia del patrimonio geo-paleontológico, y su preservación.

\section{Discusión}

Algunos de los elementos centrales de las universidades emprendedoras se reflejan en las actividades de vinculación, extensión y trabajo social que se vienen desarrollando en Paleontología y en Geología (UNRN). Justamente estas acciones pretenden generar dos cuestiones que las caracterizan. En coincidencia con 
Tarapuez Chamorro et al. (2012: 111), son: «un conjunto de mecanismos que relacionan la universidad con el entorno que la circunda» y «un grupo de docentes preparados académicamente y motivados para generar iniciativas que satisfagan las necesidades del entorno». En las experiencias desarrolladas los docentes participantes y las autoridades y el personal de los museos tenían claro que se diagnosticaban problemas en busca de soluciones con fundamentos técnicos y científicos, y paulatinamente los estudiantes asumieron que no era simplemente una práctica vinculada a contenidos de aprendizaje, sino que había problemas y otros surgían a medida que se avanzaba. Algunos de los participantes, al concluir estas, expresaron durante un grupo focal (2016-2018): «El museo se encontraba con muchas falencias que pudieron ser solucionadas, o encaminadas en otros casos»; "Parecía que los problemas no se terminaban nunca porque siempre surgían nuevos, por eso nos quedamos muchos meses más colaborando cuando ya había terminado el proyecto»; «Creo que por primera vez me di cuenta de que lo que estudiaba y lo que iba a ser cuando fuera profesional tenía mucho que ver con la sociedad, yo estudio paleontología»; «No creía que lo mío podía tener que ver con las necesidades de una escuela primaria o secundaria». Asimismo, en las encuestas a los estudiantes, el máximo valor (rango «entre 80 y 100») se da para dos pilares importantes en estas experiencias: el compromiso que les generó la integración a instituciones de la sociedad y la profundización de los aprendizajes de sus carreras. Un aspecto no considerado inicialmente, pero que los estudiantes expresaron, fue lograr un mejor aprendizaje porque conocieron a los docentes en un comportamiento diferente, que generaba un mejor clima para aprender (notas de campo registradas por las docentes-investigadoras, 2016-2018).

Para lograr resultados más satisfactorios, los equipos debieron avanzar en modificaciones, además de las normativas que fue generando la universidad. Es decir, comenzaron a sufrir procesos de cambios al interior de la propia carrera o de ciertas cátedras. En un principio no primó la reflexión ni la selección y construcción de marcos teóricos, predominando la idea de que la universidad le ofrecía algo al medio y no también a la inversa, posición predominante antes de esclarecer la «tercera misión» de las universidades (Bueno Campos, 2009; Bas, 2017; Kagan y Diamond, 2019). De ahí que no se incorporara a los diseños curriculares.

Transcurrido los primeros años, estas actividades de vinculación y extensión asumieron mayor relevancia a nivel de la universidad a medida que sus planteos eran más elaborados y mejor diseñados los planes de trabajo, y se iba comprendiendo cómo se producían los cambios hacia el interior del ámbito universitario a nivel de los aprendizajes, la docencia e, inclusive, la 
Talevi, Marianella; et al. I Prácticas de innovación pedagógica, vinculación científica-tecnológica...

investigación. En concordancia con lo que reconoce Tarapuez Chamorro et al. (2012: 110) en diversas universidades del mundo en donde:

para insertarse en dicho entorno, la Universidad Emprendedora realiza modificaciones en su cultura y en su estructura organizacional, con el ánimo de generar una respuesta más proactiva, innovadora y ágil, que dé origen a una diversidad de líneas de estudio, nuevas áreas de investigación, nuevos centros de investigación y otras instancias que gestionan la relación entre la universidad, la economía y la sociedad.

En la vinculación entre la universidad y la sociedad cabe resaltar la construcción de ciudadanía desde el enriquecimiento de los procesos de alfabetización científico-tecnológica y educación científica, aquello que Fenshan (1985) popularizó con la idea de «ciencia para todos». Algunas docentes de escuelas primarias y secundarias, al referirse al estado anterior a los textos de divulgación, la cartelería explicativa y el material audiovisual preparado para los museos en los que se trabajó, afirmaron que: «No había buenas explicaciones de lo paleontológico»; «No se enseñan estos temas vinculados a las colecciones geológicas y paleontológicas de los museos porque no se contaba con material escrito u audiovisual»; "Ahora se podrán enseñar nuevos temas que tienen que ver con la región»; y, en un sentido más general, que «El público solo mira pero no aprende tanto» (expresiones registradas en notas de campo por las docentes-investigadoras 2017-2018). Partiendo de la conceptualización de alfabetización científica de la Asociación Americana para el Avance de la Ciencia, el eje central son las interacciones entre: ciencia, tecnología, personas y sociedad; por lo tanto, el reto es la capacitación para esa interacción, que siempre será contextualizada en lo personal y social para reconocer e incidir en la resolución de los problemas que afectan la vida cotidiana (Pérez de Landazábal et al., 2011).

En los términos planteados por Cajas (2001: 243), «la falta de conocimiento tecnológico útil para que los estudiantes y futuros ciudadanos comprendan y transformen la realidad que les rodea es parte de una problemática mayor llamada bajo nivel de alfabetización científica y tecnológica». Asimismo asegura que: «Aunque alrededor del mundo existen diferentes orientaciones sobre alfabetización científica, la mayoría de las que han sido informadas no le dan a la tecnología, como conocimiento para todos, el lugar que las condiciones sociales del mundo moderno requieren» (Cajas, 2001: 243). En los últimos años, la concepción de alfabetización científico-tecnológica reconoce tanto la necesidad de llegar a todos como «que los estudiantes y adultos de 
una sociedad comprendan que al diseñar artefactos estos diseños pueden resolver problemas pero al mismo tiempo crear nuevos problemas» (Cajas, 2001: 252).

La UNRN, al ser una institución creada en la segunda década del siglo XXI, ya expresa en sus lineamientos institucionales, estratégicos y programáticos hacia el interior y el exterior los avances en torno a la relación entre universidad, alfabetización científica y ciudadanía producidos desde la década de 1980 en adelante. Muchas de sus políticas de vinculación tecnológica y extensión se relacionan con el bajo nivel de alfabetización científico-tecnológica que se observa en la región, tanto en las instituciones educativas de donde egresan los estudiantes que ingresan a la UNRN como en el personal de diversas instituciones intermedias y empresas (e.g. policía, museos y producción frutícola). Esto incide en ciertas decisiones de gestión como la creación de carreras o el rediseño de los planes de estudios. Se observan proyectos de vinculación, extensión, voluntariado y trabajo social, con planteos híbridos, superposiciones o fronteras difusas.

Si se entiende por vinculación tecnológica una forma de articular la universidad y la ciencia con distintas reparticiones del Estado, y con diversos sectores productivos y sociales, con criterios de RSU, entonces las acciones de extensión, voluntariado y trabajo social que se realizaron desde Geología y Paleontología también deben considerarse vinculación. Así se observó también en otras universidades argentinas, como lo muestran Di Bello et al. (2018) refiriéndose a la función de la vinculación como extensión (cooperación). Según Campos et al. (2005: 3), esto se origina en «la carencia de una teoría de la vinculación, y por ende, su confusión con otras actividades, básicamente las de extensión y las de prestación de servicios».

La alfabetización científico-tecnológica y la educación científica, y de la formación cívica, se fueron replanteando en las actividades de vinculación en la UNRN. Santos Rego et al. (2010) usan el concepto de «valores cívicos-profesionales» en la formación universitaria, que implica la inserción de los estudiantes en el espacio público, de ahí el reconocimiento al aprendizaje-servicio. Los participantes de las experiencias analizadas no remiten directamente a estos conceptos, si bien quedan referenciados de manera indirecta a través de los beneficios de cuestiones como que «la comunicación y vínculo creado entre varias instituciones fue muy positivo» (Entrevista a R. Barbieri, 2012); "La experiencia fue muy positiva ya que, entre otras cosas, fue una vía para conectar estudiantes universitarios con aquellos de escuelas medias» (Entrevista a S. Casadío (2012). Asimismo, lo expresaron los docentes de nivel primario y medio, desde una actitud de sorpresa y a la vez 
agradecimiento: «Me pareció muy valioso ayudar desde las universidades a este tipo de instituciones (...), justo lo que necesitábamos como transposición didáctica, ya que no tenemos nada de esto escrito para las escuelas» (Entrevista a docente, 2013).

El avance del aprendizaje-servicio fortalece una pedagogía que refuerza las competencias y facilita lo experimental (Sotelino Losada, Santos Rego y Lorenzo Moledo, 2016). Y es de esta manera que lo valoró el coordinador de la Licenciatura en Geología (UNRN), Dr. S. Casadío, ante este tipo de proyectos al ser entrevistado: «Fue de gran importancia ya que los estudiantes de Geología y Paleontología de la UNRN tuvieron una buena oportunidad de hacer prácticas» (Entrevista a S. Casadío, 2012). El replanteo también es metodológico, ya que esta enseñanza universitaria requiere diseños didácticos que den la oportunidad a que el estudiantado, con la orientación docente, identifique el problema en la comunidad, haga los diagnósticos y plantee estrategias y prácticas para su resolución. Este cambio metodológico en la enseñanza universitaria, según el mismo entrevistado, constituye para estas carreras experiencias innovadoras en la formación académica de los estudiantes, que generan «un nexo importante, ya que la conexión entre estudiantes de diferentes niveles estimula el interés por el conocimiento» (Entrevista a S. Casadío, 2012). De igual manera se expresó el director del Museo «Carlos Ameghino» (Cipolletti, provincia de Río Negro), Dr. R. Barbieri, quien además era docente en las carreras de Geología y Paleontología, destacando que «el trabajo de los estudiantes, en cuanto al ordenamiento de las colecciones, ha sido destacado», así como «muy importante para el museo, y también en la formación académica y profesional de ellos» (Entrevista a R. Barbieri, 2012).En los debates teóricos, Uribe (2018) planteó que si el foco está puesto en dar servicios solidarios (e.g. voluntariados) y no en el aprendizaje de los estudiantes, no debería considerarse aprendizaje-servicio. Finalmente, el planteo del aprendizaje-servicio demanda cambios en la cultura universitaria y organizacional, siendo central proyectar hacia el interior del planteo curricular los aprendizajes que se realizan en este tipo de acciones sociales (diseño pedagógico y didáctico, evaluación y acreditación), que es a lo que se avanza en la UNRN, en coincidencia con Kagan y Diamod (2019), que las universidades deben convertirse en «organizaciones reflexivas». Esta tensión entre las innovaciones pedagógicas de las experiencias realizadas y las instituciones se observa en algunas expresiones de los coordinadores o directores de las carreras y los directores de los proyectos, las que afloran incluso por la necesidad de financiamiento y mayor autonomía para los docentes, con expresiones como: 
Talevi, Marianella; et al. I Prácticas de innovación pedagógica, vinculación científica-tecnológica...

En mi opinión, los proyectos deberían contar con mayor apoyo financiero y ser menos burocrática su administración (...) dar mayor libertad y comenzar a confiar más en la responsabilidad de los docentes. Muchos docentes con excelentes ideas para extensión no presentan proyectos para no lidiar con la burocracia de los mismos (Entrevista a S. Casadío, 2012).

Esta posición es reforzada por otro entrevistado: «Todo lo que pueda ser mejorado en desburocratizar la implementación de estos proyectos redundará en una mayor creatividad y libertad de expresión de los participantes» (Entrevista a R. Barbieri, 2012).

\section{Conclusiones}

A través de la política institucional de vinculación y extensión implementada por la UNRN se viene consolidando, mejorando y fortaleciendo la relación entre la comunidad académica y los diversos sectores de la sociedad, con el propósito de dar respuestas a necesidades específicas locales o regionales, sin que ello implique eliminar las tensiones y las fallas que los proyectos de este tipo generan en las universidades.

Las conclusiones y reflexiones presentadas en este trabajo se agruparon en tres cuestiones: los procesos educativos de los estudiantes universitarios participantes; los cambios de la cultura universitaria y en las prácticas docentes, lo curricular e institucional, y la vinculación científica-tecnológica que involucra a la universidad con otras instituciones y la conecta a problemas específicos del contexto.

En cuanto a la primera cuestión, con estos proyectos se enriqueció la formación académica de los estudiantes y su compromiso ciudadano, poniendo a disposición de la comunidad sus saberes paleontológicos y geológicos. Los estudiantes ampliaron y profundizaron los conocimientos adquiridos en el ámbito académico a través de problemas reales en contextos específicos locales y regionales, convirtiéndose una institución externa a la universidad en un espacio alternativo de aprendizaje.

Lo mencionado es fundamental, ya que el tipo de prácticas realizadas, la aplicación de técnicas estudiadas y el acceso a numerosos materiales que son objetos de estudio se ven limitados en las universidades en relación con las necesidades de la enseñanza-aprendizaje. Asimismo, se observó que a través de las experiencias de voluntariado y trabajo social se generó un fuerte compromiso de los estudiantes, no solo con el conocimiento científico y con su formación profesional, sino con las necesidades reales del territorio. Esto 
deriva en distintos niveles de reflexión y responsabilidad que trasciende el alcance institucional de los proyectos desarrollados, pero los mismos constituyeron un claro incentivo.

En cuanto a la segunda cuestión, desde el punto de vista docente y curricular de la educación universitaria puede afirmarse que entre las primeras experiencias en 2010 y las más actuales, se avanzó en la modificación de la cultura universitaria y organizacional al incorporar este tipo acciones como parte de los procesos curriculares de aprendizaje, y en su reconocimiento para la acreditación de los saberes. Estos cambios muestran la riqueza que ofrece la teoría del aprendizaje-servicio, aun cuando la profundización del conocimiento y aplicación de la misma sigue requiriendo mayor atención y reflexión por parte de los docentes que se comprometen con estos proyectos.

Finalmente, los trabajos realizados permiten reflexionar acerca de las particularidades de la vinculación científica-tecnológica cuando no son con empresas y sus requerimientos derivados de necesidades e intereses económicos y de lucro, lo que constituye la vinculación más tradicional.

\section{Agradecimientos}

Agradecemos los comentarios y sugerencias realizadas por los revisores, que mejoraron el trabajo.

\section{Referencias bibliográficas}

ALCÁNTAR, V. M., y Arcos, J. L. (2004). La vinculación como instrumento de imagen y posicionamiento de las instituciones de educación superior. Revista Electrónica de Investigación Educativa, 6(1). Disponible en: $\quad$ http://redie.uabc.mx/vol6nol/contenido-enriquez.html

ARIAS PÉREZ, J., y Aristizábal Botero, C. (2011). Transferencia de conocimiento orientada a la innovación social en la relación ciencia-tecnología y sociedad. Pensamiento \& Gestión, (31), 137-166. Disponible en: https://www.redalyc.org/ pdf/646/64620759008.pdf
BAS, N. (2017). Las universidades y la misión de la vinculación en el Reino Unido: un marco de referencia para pensar la propuesta de indicadores del Manual de Valencia. Revista Iberoamericana de Ciencia, Tecnología y Sociedad, 12(34), 95-110.

BLANCO LÓPEZ, A. (2017). Relaciones entre la educación científica y la divulgación de la ciencia. Eureka, 1(2), 70-86. Disponible en: https://revistas.uca.es/index.php/eureka/article/view/3959

BUENO CAMPOS, E. (2007). La tercera misión de la universidad: El reto de la Transferencia del conocimiento. Revista madri+d, 41(2). 
BRUNO, D. P. (2016). Curricularizar la extensión para integrar y territorializar la práctica universitaria. Cuadernos de $\mathrm{H}$ ideas, 10(10). Disponible en: http://perio.unlp.edu. ar/ojs/index.php/cps/article/view/3727/3112

CAJAS, F. (2001). Alfabetización científica y tecnológica: La transposición didáctica del conocimiento tecnológico. Enseñanza de las Ciencias: Revista de Investigación y Experiencias Didácticas, 19(2), 243-254. Disponible en: https://www.raco.cat/index.php/ Ensenanza/article/download/21737/21571

CAMPOS, G. y Sánchez Daza, G. (2005). La vinculación universitaria: Ese oscuro objeto del deseo. Revista Electrónica de Investigación Educativa, 7(2), 13 p. Disponible en: http://redie.uabc.mx/vol7no2/contenido-campos.html

CAPRIOtTI Peri, P., y Schulze Lepe, F. (2010). Responsabilidad Social Empresarial. Colección Libros de la Empresa.

D'ANDREA, R.; Zubiría, A., y Sastre, P. (2012). Reseña histórica de la extensión universitaria. Secretaría de la Universidad Nacional del Centro de la Provincia de Buenos Aires, Buenos Aires, Argentina.1-12.

DI BelLo, M., y Romero, L. A. (2018). Vinculación y extensión universitaria: la relación entre la universidad y sus entornos nacionales en Quilmes y Lanús. Apuntes, 82, 145-171. DOI: https://doi.org/10.21678/ apuntes.82.867

FENSHAM, P. (1985). Science for all: a reflexive essay. Journal of Curriculum Studies, 17(4), 415-435.

GAETE, R. (2011). La responsabilidad social universitaria como desafío para la gestión estratégica de la Educación Superior: el caso de España. Revista de Educación, 355, 109-
133. Disponible en: http://www.revistaeducacion.educacion.es/re355/re355 05.pdf

GiBBONS, M.; Limoges, C.; Nowotny, M.; Schwartzman, S.; Scott, P., y Trow, M. (1997). La nueva producción del conocimiento. La dinámica de la ciencia y la investigación en las sociedades contemporáneas. Barcelona: Ediciones Pomares-Corredor.

HATCHER, J. A.; Bringle, R.G., y Hahn T. W. (eds.) (2017). Research on Student Civic Outcomes in Service Learning. Conceptual Framework and Methods. Virginia: Stylus Publishing.

KABABE, Y. (2010). Las unidades de Vinculación Tecnológica y la articulación entre el sector científico tecnológico y el sector empresario. SaberEs, 2, 41-58. Disponible en: https://www.saberes.fcecon.unr.edu.ar/ index.php/revista/article/view/34/66

KAGAN, C. y Diamond, J. (2019). University Community Relations in the UK. Engaging Universities. Londres: Palgrave Macmillan.

NefFA, J. C. (2000). Las innovaciones científicas y tecnológicas: Una introducción a su economía política. Buenos Aires: Editorial Lumen Humanitas.

PÉReZ de LANDAZÁBAL, M. C.; Caldeira, H., y Otero Gutiérrez, J. (2011). La relevancia de la educación científica. Madrid: Universidad Nacional de Educación a Distancia.

PUGLIESE, J. C. (2014). Políticas para el mejoramiento de la calidad. Situación actual y prospectiva. En Marquis, C. (ed.), La agenda universitaria II. Propuestas de políticas públicas para la Argentina, 155-1843. Buenos Aires: Universidad de Palermo.

RIVAS, G.; Rovira, S., y Scotto, S. (2014). Reformas a la institucionalidad de apoyo a la innovación en América Latina: antecedentes y lecciones de estudios de caso. En Rivas, 
G. y Rovira, S. (eds.), Nuevas instituciones para la innovación: Prácticas y experiencias en América Latina, 11-34. Santiago de Chile: Comisión Económica para América Latina y el Caribe (CEPAL).

SANTOS ReGo, M. A., y Lorenzo Moledo, M. M. (2010). La dimensión cívica en el desarrollo formativo de los estudiantes universitarios. Revista Electrónica de Investigación Educativa [Número Especial]. Disponible en: http://redie.uabc.mx/NumEsp2/contenido-rego.html

SOTELINO LOSADA, A.; Santos Rego, M. y Lorenzo Moledo, M. (2016). Aprender y servir en la universidad: una vía cívica al desarrollo educativo. Teoría de la Educación. Revista Interuniversitaria, 19, 225-248. Disponible en: https://revistas.usal.es/index.php/11303743/article/view/14060

SUÁREZ, R.; Roberts, J.; Voglino, S.; Diez, M. A.; González, P. y Cábana, M. C. (7-11, agosto, 2017). Rocas y minerales de Río Negro: Un espacio virtual de educación científica. XX Congreso Geológico Argentino, Geología: Presente y Futuro, Tucumán.

TARAPUez ChamorRo, E.; Osorio Ceballos, H., y Parra Hernández, R. (2012). Burton Clark y su concepción acerca de la universidad emprendedora. Tendencias, 13(2), 103-118. Disponible en: https://revistas.udenar.edu. co/index.php/rtend/article/view/474

TAYLOR, S. J. y Bogdan, R. (1996). Introducción a los métodos cualitativos de investigación. Barcelona: Paidós.

UNIVERSIDAD NACIONAL DE Río NEGRO (2009). Proyecto institucional. Parte general (ME, Res. 252/09). Disponible en: http:// repositorio.educacion.gov.ar/dspace/handle/123456789/79326
UNIVERSIDAD NACIONAL DE Río NEgRO (2015). Plan estratégico 2015-2025 de Geología (CONEAU, Res. 284/16). Disponible en:

https://www.unrn.edu.ar/archivos/ planes/21/RES\%20284\%2016\%20 CONEAU \% 20 LIC $\% 20$ EN \% 20 GEOLOGIA \%20\%20UNRN\%20SEDE\%20 AVyVM.pdf?v=1564591814

UNIVERSIDAD NACIONAL DE RÍO NEgRo (2016a). Autoevaluación de la función I+D+i 2009/2015. Disponible en: https:// www.unrn.edu.ar/downloads/plan-estrategico/Evaluaci\%C3\%B3n Funci\%C3\%B3n IDi 22 1.pdf

UNIVERSIDAD NACIONAL DE RÍO NEGRO (2016b). Autoevaluación institucional 2009-2015. Disponible en: https://www.unrn.edu.ar/section/30/evaluacion-institucional.html

UNIVERSIDAD NACIONAL DE Río NEGRO (2018a). Estatuto. Disponible en: https://www.unrn. edu.ar/images/section/descargas/115-286estatuto--de-la-universidad--nacional--derio-negro.pdf

UNIVERSIDAD NACIONAL DE Río NegRo (2018b). Reglamento de los Programas de Trabajo Social (CSDEyVE, Res. 006/2018). Disponible en: https://rid.unrn.edu.ar/bitstream/20.500.12049/1888/1/D\%202018\%20 -\%2006\%20R.pdf

URIBE, P. A. (2018). Percepción de los estudiantes de educación inicial frente al desarrollo de experiencias formativas en modalidad A+S. Revista Electrónica de Investigación Educativa, 20(4), 110-122. DOI: https://doi. org/10.24320/redie.2018.20.4.1826

WATSON, D.; Hall, L. y Tazzyman, S. (2016). Trick or treat: Academic buy-in to third stream activities. Industry and Higher Education, 30(2), 155-167. 